\title{
Ulnar Abutment Syndrome
}

\author{
Hooman Shariatzadeh ${ }^{1, *}$ \\ ${ }^{1}$ Bone and Joint Reconstruction Research Center, Shafa Orthopedic Hospital, Iran University of Medical Sciences, Tehran, IR Iran \\ *Corresponding author: Hooman Shariatzadeh, Bone and Joint Reconstruction Research Center, Shafa Orthopedic Hospital, Iran University of Medical Sciences, Tehran, IR Iran. Tel \\ +98-2133542010, Fax: +98-2133542020, E-mail: shariatzadeh_h@hotmail.com
}

Received: April 15, 2015; Accepted: May 11, 2015

Keywords: Intraosseous Ganglion; Pain; Ulnar Variance

\section{Dear Editor,}

With regard to the published article "arthroscopic curettage of intraosseous ganglions of lunate bone" in the last journal edition, it is time to review ulnar abutment syndrome with similar clinical and radiologic findings (1).

The main cause of this syndrome is the overload of carpal bones, especially lunate bone related to positive ulnar variance. In normal wrist, $20 \%$ of the load is transmitted to ulna whereas $2 \mathrm{~mm}$ positive ulnar variance increases this load up to $40 \%$. This problem may even happen in normal wrists because of ulnar variance changes during forearm rotation (2). Other predisposing factors are radial shortening after healed distal radius fractures, scapholunate dissociation, lunatotriquetral dissociation and triangular fibrocartilage complex (TFCC) tears.

Most of these patients have pain in ulnar side of wrist associated with dorsoulnar wrist tenderness and positive Nakamura's ulnar stress test (ulnar side wrist pain during flexion and extension of ulnar deviated and pronated and axially loaded wrist).

Differential diagnoses are lunate ganglion cysts, vascular grooves and Kienböck's disease.

Routine X-ray and MRI are useful for diagnosis. Radiologic findings are positive ulnar variance associated with lunate sclerosis and sometimes lunate cysts. Pronated grip view may better define ulnar impactions. Diagnostic MRI findings are TFCC degenerative tears and lunate chondromalacia.

First line of treatment is nonoperative, including decreasing wrist load and using pain killers. In the absence of good response, operative treatment may be indicated. The most prevalent surgical treatment is ulnar shortening osteotomy to unload lunate bone (3). Alternative option is wafer distal ulna resection (removal of $2-4 \mathrm{~mm}$ of distal ulna below TFCC). In Palmers types 2a through 2D, (4) salvage procedures are distal ulna resection in low demand patients (Darrach procedure), Sauve-Kapandji procedure in manual laborers, distal ulnar hemi-resection arthroplasty, and finally ulnar head replacement.

\section{References}

1. Kamrani RS, Zanjani LO, Nabian MH. Arthroscopic curettage of intraosseous ganglion of the lunate bone. Shafa Ortho J. 2014 ; 1(4):e24627

2. Palmer AK, Werner FW. The triangular fibrocartilage complex of the wrist--anatomy and function.J Hand Surg Am. 1981;6(2):153-62.

3. Constantine KJ, Tomaino MM, Herndon JH, Sotereanos DG. Com parison of ulnar shortening osteotomy and the wafer resection procedure as treatment for ulnar impaction syndrome. J Hand Surg Am. 2000;25(1):55-60.

4. Chun S, Palmer AK. The ulnar impaction syndrome: follow-up of ulnar shortening osteotomy.J Hand Surg Am. 1993;18(1):46-53. 